\title{
SUSY Particles Searches at LEP and Interpretations within the MSSM
}

\author{
Giacomo Sguazzoni ${ }^{\mathrm{a}}$ \\ aEuropean Laboratory for Particle Physics (CERN), CH-1211 Geneva 23, Switzerland
}

\begin{abstract}
Searches for R-parity conserving supersymmetric particles have been performed in $\mathrm{e}^{+} \mathrm{e}^{-}$data collected by LEP detectors, at centre-of-mass energies up to $209 \mathrm{GeV}$, corresponding to an integrated luminosity of $\sim 3.1 \mathrm{fb}^{-1}$. The results and their interpretation in the context of MSSM frameworks are briefly reviewed.
\end{abstract}

\section{INTRODUCTION}

Theories with Supersymmetry (SUSY) are the most promising extensions of the Standard Model (SM) [1]. The simplest version is the Minimal Supersymmetric Model (MSSM), which contains the minimal number of additional particles. The scalar fermions or sfermions, $\tilde{\mathrm{f}}_{\mathrm{L}}$ and $\tilde{f}_{R}$, are the partners of the left- and right-handed SM fermions and mix to form the mass eigenstates. The mixing angle $\theta_{\tilde{\mathrm{f}}}$ is so defined that $\tilde{\mathrm{f}}=\tilde{\mathrm{f}}_{\mathrm{L}} \cos \theta_{\tilde{\mathrm{f}}}+\tilde{\mathrm{f}}_{\mathrm{R}} \sin \theta_{\tilde{\mathrm{f}}}$ is the lightest sfermion. In general mixing is relevant for the third family, while $\tilde{\mathrm{f}} \equiv \tilde{\mathrm{f}}_{\mathrm{R}}$ otherwise. The SM gauge boson states have fermionic super-partners or gauginos. The MSSM higgses are arranged into two doublets and their fermionic super-partners are the higgsinos. Neutral gauginos and higgsinos mix into four mass eigenstates, the neutralinos $\chi, \chi_{2}$, $\chi_{3}, \chi_{4}\left(M_{\chi_{4}}>M_{\chi_{3}}>M_{\chi_{2}}>M_{\chi}\right)$. The charged gauginos and higgsinos mix into two mass eigenstates, the charginos $\chi^{ \pm}$and $\chi_{2}^{ \pm}\left(M_{\chi_{2}^{ \pm}}>M_{\chi^{ \pm}}\right)$.

The lepton and baryon number conservation translates into the "R-parity" conservation. The LSP (Lightest Supersymmetric Particle) is stable and must be also neutral and weakly interacting to fit the cosmological observations. Within the MSSM the LSP is the lightest neutralino $\chi$ or, less likely, the sneutrino, $\tilde{\nu}$. At LEP the sparticles are pair produced and the decay brings to final states containing at least one LSP.

The success of LEP searches is also due to the impressive performance of the accelerator that provided $\mathrm{e}^{+} \mathrm{e}^{-}$collisions at centre-of-mass energies between 161 and $209 \mathrm{GeV}$, and an integrated luminosity of $\sim 775 \mathrm{pb}^{-1}$ per experiment. The results, based on the full high-energy data sample, are presented in the form of $95 \%$ C.L. exclusion domains in the space of the relevant parameters, since no excess has been observed. When available, the LEP SUSY Working Group combinations, based on the outcomes from ALEPH, DELPHI, L3 and OPAL (ADLO), are reported [2].

\section{PRIMARY SIGNALS}

Except few pathological cases, sparticle pair production leads to the typical acoplanar particles topology due to missing energy $(\not E)$ and momentum $(\not P)$ from escaping LSP's. The energy of the visible system is related to the mass difference between the sparticle $\widetilde{\mathrm{P}}$ and the LSP $\left(\Delta M=M_{\widetilde{\mathrm{P}}}-M_{\mathrm{LSP}}\right)$. The acoplanar topologies studied cover each type of visible final state (leptons, hadronic jets, $\gamma$ 's).

\subsection{Acoplanar leptons}

The analyses for slepton signals $\left(\mathrm{e}^{+} \mathrm{e}^{-} \rightarrow \tilde{\ell}^{+} \tilde{\ell}^{-}\right.$, $\tilde{\ell} \rightarrow \ell \chi)$ search for acoplanar leptons by using the powerful lepton and tau identification of LEP detectors [3. t. $^{5} \cdot 6$. The LEP combined cross section upper limits range from 10 to $60 \mathrm{fb}$ [2]. The resulting mass lower limits are $100 \mathrm{GeV} / c^{2}$, $94 \mathrm{GeV} / c^{2}$ and $86 \mathrm{GeV} / c^{2}$ for $\tilde{e}_{\mathrm{R}}, \tilde{\mu}_{\mathrm{R}}$ and $\tilde{\tau}_{\mathrm{R}}$ respectively, valid for $\Delta M>10 \mathrm{GeV} / c^{2}$, as shown in Figure 1 .

\subsection{Acoplanar jets}

The production of a squark pair results into an acoplanar jet topology. These hadronic events can be selected by using event variables and re- 


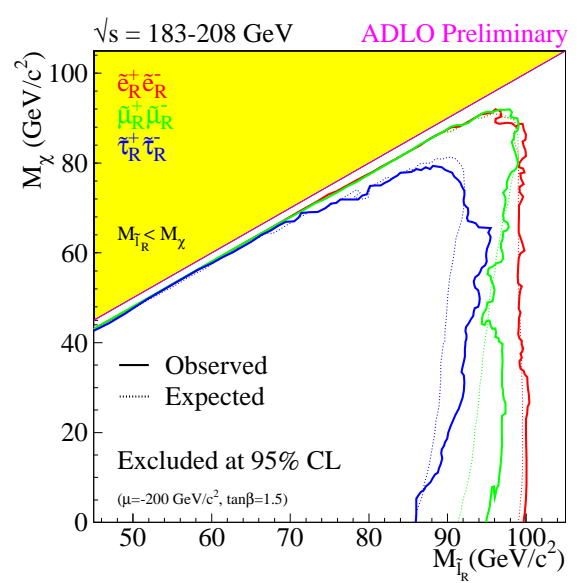

Figure 1. Slepton mass exclusion plot from the LEP SUSY Working Group.

quiring $E$ and $\not P$ 国, 5, 7, 8]. In case of $\mathrm{e}^{+} \mathrm{e}^{-} \rightarrow \tilde{\mathrm{t}} \overline{\mathrm{t}}$, $\tilde{\mathrm{t}} \rightarrow \mathrm{c} \chi$, the mass lower limit is $94 \mathrm{GeV} / c^{2}$ for $\Delta M>10 \mathrm{GeV} / c^{2}$ and any mixing, as visible in Figure 2. Further specialized selections are used for other squark processes: b-tagging is effective for $\mathrm{e}^{+} \mathrm{e}^{-} \rightarrow \tilde{\mathrm{b}} \tilde{\mathrm{b}}, \tilde{\mathrm{b}} \rightarrow \mathrm{b} \chi$, allowing a limit of $92 \mathrm{GeV} / c^{2}$ to be set $\left(\Delta M>10 \mathrm{GeV} / c^{2}\right.$, any $\left.\theta_{\tilde{\mathrm{b}}}\right)$; leptons are required in case of $\mathrm{e}^{+} \mathrm{e}^{-} \rightarrow \tilde{\mathrm{t}} \overline{\mathrm{t}}, \tilde{\mathrm{t}} \rightarrow$ $\mathrm{b} \ell \tilde{\nu}$, leading to a mass lower limit of $95 \mathrm{GeV} / c^{2}$ $\left(\Delta M>10 \mathrm{GeV} / c^{2}\right.$, any $\left.\theta_{\tilde{\mathrm{t}}}\right)$. The stop decay $\tilde{\mathrm{t}} \rightarrow \mathrm{b} \chi \mathrm{f}_{\mathrm{u}} \overline{\mathrm{f}}_{\mathrm{d}}$, recently recognized as relevant, leads to a multi-body final state topology addressed by a dedicated ALEPH selection [7. As an example, assuming the decay $\tilde{\mathrm{t}} \rightarrow \mathrm{b} \chi \mathrm{W}^{*}$, the result is $M_{\tilde{\mathrm{t}}}>77 \mathrm{GeV} / c^{2}\left(\Delta M>10 \mathrm{GeV} / c^{2}\right.$, any $\left.\theta_{\tilde{\mathrm{t}}}\right)$. ALEPH analyses also consider the case in which a stop quasi-degenerate with the LSP acquires a sizeable lifetime and hadronizes [9]. This scenario has been excluded searching for long-lived heavy hadrons and an absolute stop mass lower limit of $63 \mathrm{GeV} / c^{2}$ has been set for any $\theta_{\tilde{\mathrm{t}}}$, any branching ratio and any $\Delta M[$ [7.

\subsection{Other topologies with $E$ and $\not P$}

Topologies with two or more visible fermions in the final state plus $\not E$ and $\not P$ are expected in case of charginos and neutralinos production औ, 5. 10,11. The processes are of the type $\mathrm{e}^{+} \mathrm{e}^{-} \rightarrow$ $\chi_{i>1} \chi$ and $\mathrm{e}^{+} \mathrm{e}^{-} \rightarrow \chi_{i>1} \chi_{j>1}$ with $\chi_{i>1} \rightarrow \chi \mathrm{ff} \overline{\mathrm{f}}$, and

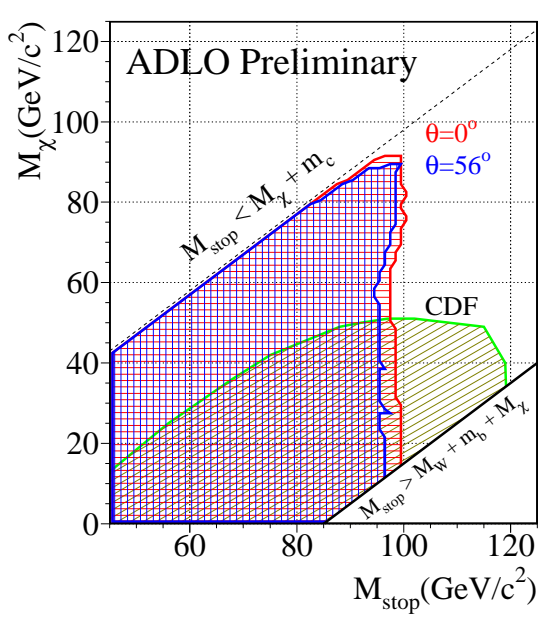

Figure 2. Stop mass exclusion plot from the LEP SUSY Working Group in case of $\tilde{\mathrm{t}} \rightarrow \mathrm{c} \chi$ decay for minimal $\left(\theta_{\mathfrak{t}}=56^{\circ}\right)$ and maximal production cross section $\left(\theta_{\tilde{\mathrm{t}}}=0^{\circ}\right)$. The CDF result is also shown.

$\mathrm{e}^{+} \mathrm{e}^{-} \rightarrow \chi^{+} \chi^{-}$with $\chi^{ \pm} \rightarrow \chi \mathrm{f}_{\mathrm{u}} \mathrm{f}_{\mathrm{d}}^{\prime}$. Cross section upper limits of $\sim 0.1-0.3 \mathrm{pb}$ are obtained by the LEP-wide outcome of dedicated selections [2].

Topologies with photon(s) can be very powerful in detecting new phenomena [5,6, 612,13$]$. Within the MSSM this case applies when heavier neutralinos are assumed to decay radiatively: $\mathrm{e}^{+} \mathrm{e}^{-} \rightarrow \chi_{2} \chi_{2}$ and $\mathrm{e}^{+} \mathrm{e}^{-} \rightarrow \chi_{2} \chi$ with $\chi_{2} \rightarrow \chi \gamma$. In this hypotheses the cross section upper limits range between $10 \mathrm{fb}$ and $0.1 \mathrm{pb}$ depending on the process [2].

\section{INTERPRETATION}

The negative results of the search for sparticle production can be translated into constraints on the parameter space in the context of specific SUSY models. Such a method allows the exclusions to be extended to sparticles otherwise not accessible, either because invisible, as the LSP, either because too heavy to be produced [4,5, 5,10$]$.

A widely accepted framework is the constrained MSSM (CMSSM). The unification of masses and couplings at the GUT scale allow the EW scale phenomenology to be set by few parameters: $\tan \beta$, the ratio of the vacuum expectation values of the two Higgs doublets; $\mu$, the Higgs sec- 


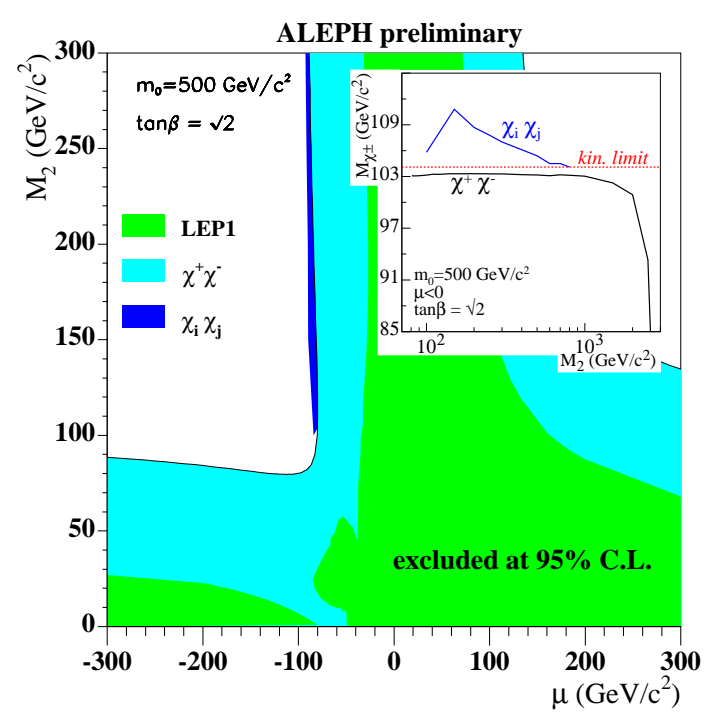

Figure 3. Excluded domains in the $M_{2}$ vs. $\mu$ plane for $\tan \beta=\sqrt{2}$ and $m_{0}=500 \mathrm{GeV} / c^{2}$. The upper-right plot shows the corresponding chargino mass lower limits for $\mu<0$.

tor mass parameter; $M_{2}$, the EW scale common gaugino mass; $m_{0}$, the GUT scale common scalar mass; the trilinear couplings $A_{\mathrm{f}}$, that enter in the prediction of the sfermion mixing and are generally set to fit the no-mixing hypothesis.

\subsection{LSP limit}

The negative outcome of charginos and neutralinos searches can be used to exclude regions in the $\left(\mu, M_{2}\right)$ plane, as shown, as an example, in Figure 3 in which the sleptons are assumed to decouple (i.e. large $m_{0}$ ). The upper-right plot of Figure 3 shows how neutralino searches allow chargino exclusions to be improved for small $\tan \beta$ and $\mu<0$. If the sleptons are lighter (small $m_{0}$ values), the chargino and neutralino cross sections decrease for the enhancement of negativeinterfering slepton-exchange diagrams. The consequent loss of sensitivity is recovered by slepton searches in such a way that lower mass limits on gauginos and other sparticles as $\tilde{e}_{\mathrm{R}}$ or $\tilde{\nu}$ could be set [4, 5, 6, 14. Among these, the most important is the LSP limit, i.e. the mass lower limit on $\chi$, shown in Figure 1 as a function of $\tan \beta$ for coupling and decoupling sleptons. The LSP

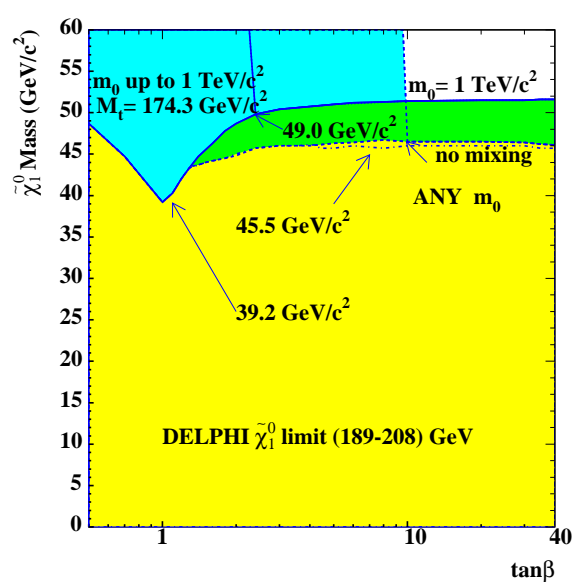

Figure 4. The $\chi$-LSP limit vs. $\tan \beta$ : large $m_{0}$ (solid curve) and any $m_{0}$ (dashed curve) in case of no-mixing; any $m_{0}$ and $A_{\tau}=A_{\mathrm{b}}=A_{\mathrm{t}}=0$, i.e. mixing in the third family (dash-dotted curve). The steep lines show the impact of Higgs boson searches for two $m_{0}$ scenarios $[$ 何.

mass lower limits from LEP experiments fall between $36.3 \mathrm{GeV} / c^{2}$ and $39.6 \mathrm{GeV} / c^{2}$ and are set for $\tan \beta \sim 1$.

The LEP mass lower limits on the Higgs boson mass $m_{\mathrm{h}}$ can be also used to further exclude small $\tan \beta$ ranges. Roughly, this just derives from the MSSM tree-level relation $m_{\mathrm{h}}<m_{\mathrm{Z}}|\cos \beta|$. However, the details of the exclusion depend on $M_{2}$, $m_{0}$ and the stop mass because of the large radiative corrections to $m_{\mathrm{h}}$. Adding the Higgs constraints the LSP mass lower limit substantially improves (up to $\sim 45 \mathrm{GeV} / c^{2}$ ) and moves towards $\tan \beta \sim 4$, as shown in Figure 4 .

\subsection{Impact of mixing in the third family}

The robustness of the LSP limit has been checked with respect to the mixing effects in the third family, neglected in the above discussion. A stau getting light for mixing may be mass degenerate with the LSP, making the chargino decays into staus difficult to detect [4] 10]. Dedicated selections for $\chi^{ \pm} \rightarrow \tilde{\tau} \nu_{\tau} \rightarrow \tau \chi \nu_{\tau}$ with soft taus, $\mathrm{e}^{+} \mathrm{e}^{-} \rightarrow \chi_{2} \chi$ and $\mathrm{e}^{+} \mathrm{e}^{-} \rightarrow \chi_{2} \chi_{2}$ with $\chi_{2} \rightarrow \tau \tau \chi$, and for chargino production in association with an ISR photon $\left(\mathrm{e}^{+} \mathrm{e}^{-} \rightarrow \chi^{+} \chi^{-} \gamma\right)$ allow to solve this problem. As shown in Figure 1 , the LSP 


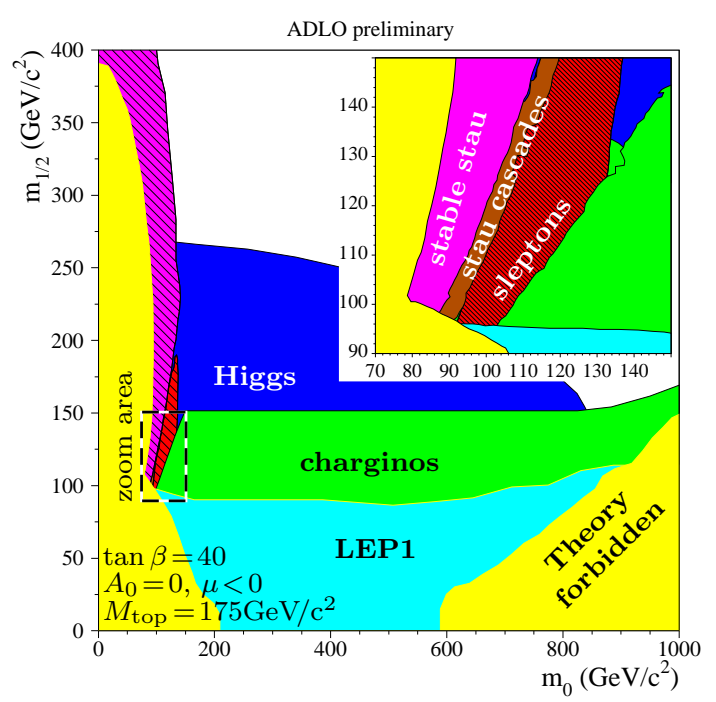

Figure 5. LEP combined exclusion domains in the mSUGRA $m_{1 / 2}$ vs. $m_{0}$ plane for $\tan \beta=40$, $A_{0}=0$ and $\mu<0$. A peculiar area is zoomed to show the interplay between selections.

limits reported above have been demonstrated to hold by using this studies, extended also considering the mixing configurations for $\tilde{\tau}, \tilde{\mathrm{t}}$ and $\tilde{\mathrm{b}}$ that can be explored by setting $A_{\tau}, A_{\mathrm{t}}$ and $A_{\mathrm{b}}$ to zero.

\section{3. mSUGRA}

The results have been also interpreted within an even more constrained version of the CMSSM, usually referred to as Minimal Supergravity (mSUGRA). The relevant parameters are: $\tan \beta$, the sign of $\mu$ and $m_{0} ; m_{1 / 2}$, the GUT scale common gaugino mass, that replaces $M_{2} ; A_{0}$, the GUT scale common trilinear coupling.

On top of LEP1 exclusions and theoryforbidden regions, small $m_{0}$ and $m_{1 / 2}$ areas are constrained from sleptons and gaugino searches, respectively. Higgs boson searches are also effective, even in the large $\tan \beta$ range. As an example, Figure 5 illustrates $m_{1 / 2}$ vs. $m_{0}$ excluded domains for $\tan \beta=40, \mu<0$ and $A_{0}=0$. The zoomed area focuses on the pathological region in which, for the mixing, $\tilde{\tau}$ and $\chi$ are almost degenerate and the selections for stau-cascades and stable staus have to be used. The resulting mSUGRA LSP mass lower limits lie between
52 and $59 \mathrm{GeV} / c^{2}$, depending on the top mass, and turn out to be $\sim 8-9 \mathrm{GeV} / c^{2}$ lower if $A_{0}$ is allowed to assume values other than zero [2].

\section{CONCLUSION}

Despite the negative outcome, LEP has substantially contributed to the study of supersymmetric scenarios. Stringent constraints on sparticle parameters have been set by direct search and by interpretation studies within widely accepted frameworks. This huge experience is an important part of the LEP legacy and it will result very useful for SUSY searches at future experiments.

\section{ACKNOWLEDGMENTS}

I would like to thank G. Ganis for the careful reading of the manuscript. Special thanks also to P. Azzurri for his kind hospital-ity.

\section{REFERENCES}

1. K. Hagiwara et al., Phys. Rev. D 66 (2002) 010001; S.P. Martin, hep-ph/9709356 (1997); N. Polonsky, hep-ph/0108236 (2001).

2. http://lepsusy.web.cern.ch/lepsusy/

3. ALEPH Collaboration, Phys. Lett. B 526 (2002) 206.

4. DELPHI Collaboration, DELPHI Note 2002027-CONF-561 (2002).

5. http://13.web.cern.ch/13/

6. OPAL Collaboration, OPAL Physics Note PN470 (2002).

7. ALEPH Collaboration, Phys. Lett. B 537 (2002) 5.

8. OPAL Collaboration, Phys. Lett. B $\mathbf{5 4 5}$ (2002) 272.

9. ALEPH Collaboration, Phys. Lett. B 488 (2000) 234.

10. ALEPH Collaboration, ALEPH/2002-028 CONF/2002-017 (2002).

11. OPAL Collaboration, OPAL Physics Note PN503 (2002).

12. ALEPH Collaboration, CERN-EP-2002-033.

13. DELPHI Collaboration, DELPHI Note 2002077-CONF-611 (2002).

14. ALEPH Collaboration, Phys. Lett. B 544 (2002) 73 . 